\title{
A study on the demographic, clinical, and radiological profile of lineage B.1.1.7 (United Kingdom) strain Covid-19 patients at Telangana institute of medical sciences and research, Hyderabad
}

\author{
Jayasri Helen Gali', Manasa Musku², Devireddy Pallavi Reddy ${ }^{3}$, Vimala Thomas ${ }^{4}$, \\ Ehsan Ahmed Khan ${ }^{5}$ \\ ${ }^{1}$ Professor and Head of Department, ${ }^{2,3}$ Assistant Professor, Department of Pulmonary Medicine, ${ }^{4}$ Professor, \\ Department of Social and Preventive Medicine and Director, ${ }^{5}$ Assistant Professor, Department of General Medicine and \\ Medical Superintendent, Telangana Institute of Medical Sciences and Research, Hyderabad, Telangana, India
}

Background: Emergence of variants with specific mutations in key epitopes in the spike protein of severe acute respiratory syndrome coronavirus 2 (SARS-CoV-2) raises concerns pertinent to its severity. Aims and Objectives: To assess the demographics, clinico-radiological pattern, and outcome of reverse transcription-polymerase chain reaction (RT-PCR) positive isolated coronavirus disease 2019 (COVID-19) patients arrived from the United Kingdom (UK). Materials and Methods: A cross-sectional, observational study was conducted in RT-PCR positive COVID-19 patients arrived from the UK, from December 2020 to February 2021. Nasopharyngeal samples of all patients were sent for whole-genome sequencing of SARS-CoV-2. The aimed parameters were compared between the B.1.17 positive and non-B.1.1.7 groups, among the people, arrived from the UK. All statistical tests with $\mathrm{P}<0.05$ were considered significant. Results: A total of 59 SARS-CoV2 infected patients, who arrived from the UK, were isolated from December 2020 to February 2021 at Telangana Institute of Medical Sciences hospital, Hyderabad, were enrolled in the study. Of these, 27 patients (mean age-31.81 \pm 11.28 years) were infected with the B.1.1.7, diagnosed by whole genomic sequencing. Males were predominant in our study. Personal habits such as smoking, alcohol intake were higher among the sequenced group with a significant $\mathrm{P}<0.05$. The most common symptoms observed in the sequenced group were cough $(22.22 \%)$, sore throat $(22.22 \%)$, cold $(11.11 \%)$, fever $(11.11 \%)$, and in the unsequenced group were cough $(22.22 \%)$, cold $(6.25 \%)$, fever $(6.25 \%)$. In the sequenced group, chest X-ray posteroanterior view was normal in $74 \%$, patchy groundglass opacities was observed in $25.92 \%$ patients, whereas in the unsequenced group it was $84.37 \%$ and $15.62 \%$, respectively. Asymptomatic patients, observed in the sequenced and unsequenced group were $48.18 \%$ and $78.12 \%$, respectively, and symptomatic patients were $51.85 \%$ and $21.87 \%$, respectively. We found a statistically significant difference between sequenced and unsequenced patients in the asymptomatic group with a significant $\mathrm{P}<0.05$. In the sequenced and unsequenced group, mild cases were $48.18 \%$ and $18.75 \%$, moderate cases were $3.7 \%$ and $3.12 \%$, respectively. We found no evidence of an association between disease severity and lineage B.1.17. Conclusion: Our data, within the context and limitations of a real-world study, provide initial reassurance that severity in hospitalized patients with B.1.1.7 is not markedly different from severity in those without B.1.1.7.

Key words: Coronavirus disease 2019; Genomic sequencing; Mutation; Reverse transcription polymerase chain reaction; Severe acute respiratory syndrome coronavirus 2 B.1.1.7; Symptom

\section{Access this article online}

\section{Website:}

http://nepjol.info/index.php/AJMS

DOI: 10.3126/ajms.v13i1.40630

E-ISSN: 2091-0576

P-ISSN: $2467-9100$

Copyright (c) 2022 Asian Journal of Medical Sciences

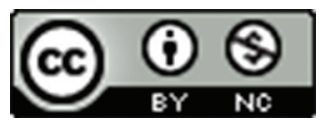

This work is licensed under a Creative Commons Attribution-NonCommercial 4.0 International License. 


\section{INTRODUCTION}

Coronavirus disease 2019 (COVID-19), the highly contagious viral illness caused by severe acute respiratory syndrome coronavirus 2 (SARS-CoV-2), has had a catastrophic effect on the world's demographics resulting in more than 3.8 million deaths worldwide, emerging as the most consequential global health crisis since the era of the influenza pandemic of 1918. After the first cases of this predominantly respiratory viral illness were first reported in Wuhan, Hubei Province, China, in late December 2019, SARS-CoV-2 rapidly disseminated across the world in a short span of time, compelling the World Health Organization (WHO) to declare it as a global pandemic on March 11, 2020. ${ }^{1}$ The disease has since spread worldwide leading to an ongoing pandemic. ${ }^{2}$ Several variants of SARS-CoV-2 have been described during the course of this pandemic, among which only a few are considered variants of concern (VOCs) by the WHO, given their impact on global public health. ${ }^{1}$ Based on the recent epidemiological update by the $\mathrm{WHO},{ }^{3}$ as of July 2021, four VOCs have been identified since the beginning of the pandemic namely, Alpha, Beta, Gamma, and Delta and the Variants of Interest are Eta, Lota, Kappa, and Lambda. ${ }^{3}$ On December 14, 2020, the United Kingdom (UK) Government was notified of the emergence of a SARSCoV-2 variant under investigation. ${ }^{4}$ This lineage had no obvious genetically close precursor within publicly available genomic datasets, within the COVID-19 Genomics UK Consortium dataset, and is now defined as lineage B.1.1.7. Defining features include a deletion and several mutations within the key encoding the spike protein, notably Asn501Tyr (N501Y) in the receptor-binding domain. The effect of structural changes to the properties of the spike protein has sparked concern about transmissibility, pathogenicity, and effect of the variant on vaccine efficacy. Physical distancing, masking was strictly implemented in London on December 21, 2020, to curb further spread of this variant. Further variants have since been reported with potentially similar properties to the B.1.1.7 variant. ${ }^{6,7}$ The impact of the change on clinical presentation, patient outcome, and mortality remains poorly understood. ${ }^{8}$ Few studies have observed that individuals infected with the variant of concern, identified at UK community centers, were between $32 \%$ and $104 \%$ more likely to die than individuals infected with previously circulating variants. ${ }^{8}$ In the current study, at our institute, we assessed the demographics, clinico-radiological pattern, and outcome of RT-PCR positive isolated COVID-19 patients arrived from UK.

\section{Aims and Objectives}

To assess the demographics, clinico-radiological pattern and outcome of RT-PCR positive isolated covid 19 patients arrived from United Kingdom.

\section{MATERIALS AND METHODS}

A cross-sectional, observational study was conducted from December 2020 to February 2021 at Telangana Institute of Medical Sciences (TIMS), a dedicated hospital for COVID-19 management. As a nodal center, it was responsible for surveillance of international travelers. The study was pre-approved by the institutional scientific committee. As per standard operating procedure (SOP) $)^{9,10}$ guidelines all the individuals traveling from abroad must undergo RT-PCR testing on their arrival at Hyderabad airport.

As per the policy of the Indian government, which is followed by the state government, all the persons traveling from the UK to Hyderabad were tested for COVID-19 by taking a nasopharyngeal swab for RT-PCR at the time of their arrival at the Airport, Hyderabad, after which they were instructed to wait for about $6 \mathrm{~h}$ in the airport for the result. Samples were taken from all persons on landing, irrespective of the status of prior testing report. The persons whose swab is detected positive for COVID-19 were isolated at dedicated floor, in separate rooms at TIMS, irrespective of their symptoms or severity. Clinical data and lab results were collected and analyzed as described below.

The demographic data such as age, sex, personal habits, occupation were noted. Clinical data such as symptoms, signs, comorbidities, vitals were taken. The patients were thoroughly examined and evaluated. Basic laboratory work up such as complete blood picture, liver and renal function tests, random blood sugar, and chest X-ray was done for all patients, additional tests such as D-dimer, lactate dehydrogenase (LDH), C-reactive protein (CRP) were done based on clinical severity and severity grading was done according to Indian Council of Medical Research (ICMR) guidelines. ${ }^{11}$ Nasopharyngeal sample of all patients were sent for whole genome sequencing for COVID-19. The turn-around time for genome sequencing is about 3-4 days, once the result is known, patients positive for lineage B.1.1.7., strain were isolated for 17 days (from time of initial swab testing for RT-PCR at arrival), persons negative for lineage B.1.1.7., strain were transferred or discharged to other floor for further treatment according to the ICMR guidelines. ${ }^{11}$ Two repeat samples $24 \mathrm{~h}$ apart were taken for patients with positive lineage B.1.1.7., after 17 days for decision on discharge. Thus, an analysis on the demographics, clinico-radiological pattern, and outcomes of the lineage B.1.1.7., strain positive persons was done. Then the demographic features, laboratory parameters, chest $\mathrm{x}$-ray findings, disease severity, and outcome were compared between the B.1.17 positive and non-B.1.1.7 groups among the people arrived from UK. 


\section{Definition}

The patients tested positive for lineage B.1.1.7 by whole genome sequencing were considered under sequenced group and those patients who tested negative for the lineage B.1.1.7 were considered unsequenced.

\section{Statistical analysis}

All the collected data were compiled and entered into Microsoft excel. Data were checked for consistency and completeness, and then analyzed using SPSS windows version 27 software. Proportions were calculated for all qualitative variables. Chi-square test was performed to study the association between two categorical variables. All statistical tests with $\mathrm{P}<0.05$ were considered significant.

\section{RESULTS}

A total of 59 SARS-CoV2 infected patients, who arrived from the UK, were isolated from December 2020 to February 2021 at TIMS hospital, Hyderabad, were enrolled in the study. Of these, 27 patients (mean age- $31.81 \pm 11.28$ years) were infected with the B.1.1.7, diagnosed by whole genomic sequencing (Table 1). The remaining 32 patients (mean age-30.9 \pm 12.14$)$ were diagnosed as non-B.1.1.7. Although males were predominant in our study (Male: Female ratio 4.4:1 vs. Male: Female ratio 2.2:1) no statistical significance $(\mathrm{P}=0.27)$ was found among both the groups (Table 1$)$.

Personal habits such as smoking (29.62\%), alcohol intake $(25.92 \%)$ was higher among the sequenced group with a significant $\mathrm{P}<0.05$. Most of the patients were students $(37.03 \%)$ and laborers $(37.03 \%)$ in the sequenced group and in the unsequenced group students (31.25\%) and IT professionals $(31.25 \%)$ were predominant (Table 1$)$. The comorbidities observed in the sequenced group were hypertension $(7.4 \%)$, type 2 diabetes mellitus $(3.7 \%)$, hypothyroidism $(3.7 \%)$, and systemic lupus erythematosus $(3.7 \%)$, whereas in the unsequenced group were, type 2 diabetes mellitus (6.25\%), hypertension $(3.12 \%)$ and obesity $(3.12 \%)$. In the sequenced group, $51.85 \%$ of patients arrived directly from the UK, $44.45 \%$ of patients had transit history. In the unsequenced group, $93.75 \%$ of patients arrived directly from the UK and $6.25 \%$ of patients had transit history (Table 1).

The most common symptoms observed in the sequenced group were cough $(22.22 \%)$, sore throat $(22.22 \%)$,

\begin{tabular}{|c|c|c|c|c|c|}
\hline Parameter & & $\begin{array}{c}\text { WGS (Lineage } \\
\text { B.1.1.7) }(n=27)(\%)\end{array}$ & $\begin{array}{l}\text { Non-WGS } \\
(n=32)(\%)\end{array}$ & $\begin{array}{l}\text { Chi-square } \\
\text { value }\end{array}$ & P-value \\
\hline \multirow[t]{2}{*}{ Age in years } & $<30$ years & $15(55.56)$ & $16(50)$ & 0.18 & 0.67 \\
\hline & $>30$ years & $12(44.45)$ & $16(50)$ & & \\
\hline \multirow[t]{2}{*}{ Gender } & Male & $22(81.5)$ & $22(68.75)$ & 1.23 & 0.27 \\
\hline & Female & $5(18.5)$ & $10(31.25)$ & & \\
\hline \multirow[t]{3}{*}{ Habits } & Smoking & $8(29.62)$ & $4(12.5)$ & 4.15 & $<0.05$ \\
\hline & Alcohol & $7(25.92)$ & $2(6.25)$ & & \\
\hline & Both & $5(18.5)$ & - & & \\
\hline \multirow[t]{4}{*}{ Occupation } & Student & $10(37.03)$ & $10(31.25)$ & 11.99 & $<0.05$ \\
\hline & Labourer & $10(37.03)$ & $2(6.25)$ & & \\
\hline & IT professional & $2(7.4)$ & $10(31.25)$ & & \\
\hline & $\begin{array}{l}\text { Others (homemaker, } \\
\text { teacher,business etc) }\end{array}$ & $5(18.5)$ & $10(31.25$ & & \\
\hline Comorbidites & Present & $4(14.81)$ & $4(12.5)$ & 0.06 & 0.79 \\
\hline \multirow{2}{*}{ Travel History } & Direct UK & $14(51.85)$ & $30(93.75)$ & 12.25 & $<0.05$ \\
\hline & Transit journey & $12(44.45)$ & $2(6.25)$ & & \\
\hline \multicolumn{6}{|l|}{ Symptoms } \\
\hline Cough & Present & $6(22.22)$ & $6(22.22)$ & 2.93 & 0.08 \\
\hline Sore throat/throat pain & Present & $6(22.22)$ & $1(3.12)$ & 5.02 & $<0.05$ \\
\hline Fever & Present & 3 (11.11) & $2(6.25)$ & 0.43 & 0.50 \\
\hline Cold & Present & $3(11.11)$ & $2(6.25)$ & 0.43 & 0.50 \\
\hline Breathlessness & Present & $1(3.7)$ & $1(3.12)$ & 0.01 & 0.90 \\
\hline \multicolumn{6}{|l|}{ Investigations } \\
\hline \multirow{2}{*}{$\begin{array}{l}\text { 1. Neutrophilic leucocytosis } \\
\text { Leucopenia }\end{array}$} & Present & $3(11.11)$ & $5(15.62)$ & 0.25 & 0.61 \\
\hline & Present & $5(18.5)$ & $1(3.12)$ & 3.73 & 0.05 \\
\hline Neutrophil lymphocyte ratio $>3.5 \%$ & Present & $1(3.7)$ & $1(3.12)$ & 0.01 & 0.90 \\
\hline 2. Chest X-ray changes & Present & $7(25.92)$ & $5(15.62)$ & 0.95 & 0.327 \\
\hline $\begin{array}{l}\text { 3. Elevated Inflammatory markers } \\
\text { (CRP) }\end{array}$ & Present & $3(11.11)$ & - & 3.68 & 0.50 \\
\hline \multirow[t]{3}{*}{ COVID-19 severity } & Asymptomatic & $13(48.18)$ & $25(78.12)$ & 5.98 & 0.05 \\
\hline & Mild & $13(48.18)$ & $6(18.75)$ & & \\
\hline & Moderate & $1(3.7)$ & $1(3.12)$ & & \\
\hline
\end{tabular}

CRP: C-reactive protein 
cold $(11.11 \%)$, fever $(11.11 \%)$, and in the unsequenced group were cough $(22.22 \%)$, cold $(6.25 \%)$, fever $(6.25 \%)$ (Table 1). The mean duration of the symptoms observed were $1.96 \pm 2.15$ days and $0.96 \pm 1.92$ days in the sequenced and unsequenced group, respectively. Complete blood examination results showed neutrophilic leucocytosis $(15.62 \%)$ more in the unsequenced group, whereas leucopenia $(18.5 \%)$ was more in the sequenced group. Neutrophil-lymphocyte ratio $>3.5$ elevation was similar in both the groups $(\mathrm{P}>0.05)$. In the sequenced group, $11.11 \%$ of patients has elevated CRP levels (Table 1). Renal and liver function tests were normal in both groups.

In the sequenced group, chest X-ray posteroanterior (PA) view was normal in $74 \%$, patchy ground-glass opacities was observed in $25.92 \%$ patients $(18.5 \%)$ patients have bilateral and $7.4 \%$ has unilateral involvement; Lower zones were predominantly involved $(22.22 \%)$ followed by mid zones $(3.7 \%)$. In the unsequenced group, chest X-ray PA view was normal in $84.37 \%$, patchy ground-glass opacities was observed in $15.62 \%$ of patients $(6.25 \%)$ patients has bilateral and $9.37 \%$ has unilateral involvement; Lower zones were predominantly involved $(12.5 \%)$ followed by mid zones $(3.12 \%)$. No statistical significance was observed among both groups $(\mathrm{P}=0.327$ ) (Table 1$)$.

Asymptomatic patients, observed in the sequenced and unsequenced group were $48.18 \%$ and $78.12 \%$, respectively, and symptomatic patients were $51.85 \%$ and $21.87 \%$, respectively. We found a statistical difference with a significant $\mathrm{P}<0.05$ between sequenced and unsequenced group. In the sequenced and unsequenced group, mild cases were $48.18 \%$ and $18.75 \%$, moderate cases were $3.7 \%$ and $3.12 \%$, respectively. None were severe in both groups; no mortality was observed and the outcome was good in both the groups. We found no evidence of an association between disease severity and lineage (B.1.1.7 vs. non-B.1.1.7) (Table 1).

\section{DISCUSSION}

The emergence of novel VOCs in the ongoing SARSCoV-2 pandemic requires rapid genomic, virological, epidemiological, and clinical characterization to ascertain public health, clinical, and research responses.

Though advancing age is associated with high mortality, the most common age group observed in our study was between 20 and 30 years, with male predominance with low incidence of comorbidities in both the groups. Although all age groups are at risk of contracting COVID-19, older people face significant risk of developing severe illness if they contract the disease due to physiological changes that come with aging and potential underlying health conditions. Therefore, less number of elderly population might have traveled during the pandemic. A study conducted by Dan Frampton et al., ${ }^{12}$ which stated Patients with B.1.1.7 were younger and had fewer comorbidities than those with non-B.1.1.7 infection, possibly representing the widespread and potential increased transmission of this variant in the community or differences in the probability of hospital admission, which we were not able to explore in this hospital-based cohort. ${ }^{12}$

Personal habits such as smoking, alcohol intake was found higher in the sequenced group, and evidence suggests that the severity of COVID-19 disease is higher among smokers as smoking impairs lung function. A UK study published in January 2021 suggests that current smokers who get coronavirus are twice as likely to attend hospital and tend to report more symptoms than non-smokers. ${ }^{13}$ Most of the population in our study were students, laborers, and IT professionals. Survey data from the UK suggest that less than one in five people are able to adhere to isolation protocols. Notably, lower rates of adherence have been reported among men, younger people, key workers, those living with dependent children, and those in lower socioeconomic groups. ${ }^{14}$

Only 1 person in the sequenced group was vaccinated, Peacock said, "With this variant, there is no evidence that it will evade the vaccination or a human immune response. But if there is an instance of vaccine failure or reinfection then that case should be treated as high priority for genetic sequencing." 4

Most of the symptoms observed in both the groups were similar. Two studies, published in Lancet Infectious Diseases ${ }^{12}$ and Lancet Public Health, ${ }^{15}$ have found no evidence that people infected with B.1.1.7 (UK variant of coronavirus) experience worse symptoms or a heightened risk of developing "long COVID" compared to those infected with a different COVID-19 strain.

In sequenced group complete blood picture showed neutrophilic leukocytosis, leukopenia, increased neutrophillymphocyte ratio (NLR) in $11.11 \%, 18.5 \%, 3.7 \%$ patients, respectively, in unsequenced patients neutrophilic leukocytosis, leukopenia, increased NLR in $15.62 \%$, $3.12 \%$, and $3.12 \%$ patients, respectively. In the sequenced group elevated inflammatory markers were present in $11.11 \%$ of patients. Inflammation is caused by infectious diseases, and growing evidence supports its significant role in the progression of various viral pneumonias, including COVID-19. Severe inflammatory responses contribute to weak adaptive immune response, thereby resulting in immune response imbalance. Therefore, 
circulating biomarkers such as peripheral white blood cell count, hemoglobin, NLR ratio, CRP that can represent inflammation and immune status are potential predictors for the prognosis of COVID-19 patients. $^{16}$

In most of our study population, chest X-ray findings were normal, about $74 \%$ in the sequenced group, $84.37 \%$ in the unsequenced group, respectively. The chest radiograph may be normal in up to $63 \%$ of people with COVID-19 pneumonia, particularly in the early stages. ${ }^{17}$ In our study, the most common change observed was patchy ground-glass opacities, distributed peripherally in bilateral lower lung zones. Earlier studies also showed ground glass appearance is common and may precede the appearance of consolidation and bilateral lung involvement. ${ }^{17}$

Although we observed a statistical difference in the symptomatology and lineage, we found no statistical significance in the disease severity and lineage. A study conducted by Davies et al., ${ }^{18}$ have established that B.1.1.7 is more transmissible than pre-existing variants, but have not identified whether it leads to any change in disease severity. A matched case-control study conducted by Benjamin et al. ${ }^{19}$ in 60 hospitalized patients, results shows a tendency towards severe disease with B.1.1.7 infection, however, the study was underpowered as statistical significance was seen only for patients requiring dexamethasone.

Rapid collection of good quality clinical data with the appropriate granularity, in combination with whole-genome sequencing of SARS-CoV-2, is imperative in deciding whether variants are associated with altered clinical outcomes. Large readily available datasets will be key in enabling rapid clinical assessment of variants.

\section{Limitations of the study}

- Our study was conducted on a smaller sample size,thus there is a need for further studies on larger sample size

- Most of the persons were sub clinical in our study, as it was done in persons fit for travel, screened incidentally for B.1.1.7 lineage. We cannot comment on persons with symptomatic forms of Covid-19, as they would restrict travel.

- We predominantly had younger age group in our study, possibly because this group of persons travel more than the other groups, especially during a pandemic when middle and older groups restricted travel.

\section{CONCLUSION}

Our data, within the context and limitations of a realworld study, provide initial reassurance that severity in hospitalized patients with B.1.1.7 is not markedly different from severity in those without B.1.1.7.

\section{ACKNOWLEDGMENT}

We thank Dr. Pravin Pisudde, Associate Professor, Department of Social and Preventive Medicine, ESI medical college and hospital, Hyderabad for helping us with stastical analysis.

\section{REFERENCES}

1. Cascella M, Rajnik M, Aleem A, Dulebohn SC and Di Napoli R. Features, evaluation, and treatment of Coronavirus (COVID-19). In: StatPearls. Treasure Island, FL: StatPearls Publishing; 2021.

2. Zimmer C. The secret life of a Coronavirus-an Oily, 100-Nanometer-Wide Bubble of Genes Has Killed more than Two Million People and Reshaped the World. Scientists Don't Quite Know What to Make of it; 2021.

3. Tracking SARS-Cov-2 Variants. World Health Organization; 2021.

4. Wise J. COVID-19: New Coronavirus variant is identified in UK. BMJ. 2020;371:m4857. https://doi.org/10.1136/bmj.m4857

5. Rambaut A, Loman N, Pybus O, Barclay W, Barrett J, Carabelli A, et al. (CoG-UK). Preliminary Genomic Characterisation of an Emergent SARS-CoV-2 Lineage in the UK Defined by a Novel Set of Spike Mutations; 2020. Available from: https://www. virological.org/t [Last accessed on 2021 Jan 31].

6. Tegally H, Wilkinson E, Giovanetti M, Iranzadeh A, Fonseca V, Giandhari J, et al. Emergence and Rapid Spread of a New Severe Acute Respiratory Syndrome-Related Coronavirus 2 (SARS-CoV-2) Lineage with Multiple Spike Proteins in South Africa, medRxiv; 2020.

https://doi.org/10.1101/2020.12.21.20248640

7. Faria $\mathrm{N}$, Claro IM, Candido D, Franco LA, Andrade PS, Coletti TM, et al. Genomic Characterisation of an Emergent SARS-CoV-2 Lineage in Manaus: Preliminary Findings; 2021. Available from: https://www.virological.org/t/genomiccharacterisation-ofan-emergent-sars-cov-2-lineage-in-manauspreliminaryfindings/586 [Last accessed on 2021 Jan 31].

8. Challen R, Brooks-Pollock E, Read JM, Dyson L, TsanevaAtanasova $\mathrm{K}$ and Danon L. Risk of mortality in patients infected with SARS-CoV-2 variant of concern 202012/1: Matched cohort study. BMJ. 2021;372:n579.

https://doi.org/10.1136/bmj.n579

9. Standard Operating Procedure for Epidemiological Surveillance and Response in the Context of New Variant of SARS-CoV-2 Virus Detected in United Kingdom; 2020. Available from: https:// www.mohfw.gov.in/pdf. [Last accessed on 2020 Dec 22].

10. Standard Operating Procedure for Epidemiological Surveillance And Response for the New Variant of SARS-CoV-2 in the Context of Regulated Resumption of Limited Flights Originating from United Kingdom (UK) to India from $8^{\text {th }}$ January, 2021; 2021. Available from: https://www.mohfw.gov.in/pdf sop. [Last accessed on 2021 Jan 30].

11. Clinical Guidance for Management of Adult COVID-19 Patients; 2021. Available from: https://www.icmr.gov.in/pdf/ covid/techdoc/covid_management_algorithm_17052021.pdf. [Last accessed 2021 May 17]. 
12. Frampton $\mathrm{D}$, Rampling $\mathrm{T}$, Cross $\mathrm{A}$, Bailey $\mathrm{H}$, Heaney J, Byott $\mathrm{M}$, et al. Genomic characteristics and clinical effect of the emergent SARS-CoV-2 B.1.1.7 lineage in London, UK: A whole-genome sequencing and hospital-based cohort study. Lancet Infect Dis. 2021;21:1-11.

https://doi.org/10.1016/S1473-3099(21)00170-5

13. Hopkinson NS, Rossi N, El-Sayed Moustafa J, Laverty AA, Quint JK, Freidin M, et al. Current smoking and COVID-19 risk: Results from a population symptom app in over 2.4 million people. Thorax. 2021;76(7):714-722.

https://doi.org/10.1136/thoraxjnl-2020-216422

14. Available from: https://www.assets.publishing.service.gov. uk/government/uploads/system/uploads/attachment_data/ file/965094/s1100covid-19riskbyoccupationworkplace.pdf. [last accessed 2021 Feb 11].

15. Graham MS, Sudre $\mathrm{CH}$, May A, Antonelli M, Murray B, Varsavsky $\mathrm{T}$, et al. Changes in symptomatology, reinfection, and transmissibility associated with the SARS-CoV-2 variant B.1.1.7:
An ecological study. Lancet Public Health. 2021;6(5):e335-e345. https://doi.org/10.1016/S2468-2667(21)00055-4

16. Yang AP, Liu JP, Tao WQ, Li HM. The diagnostic and predictive role of NLR, d-NLR and PLR in COVID-19 patients. Int Immunopharmacol. 2020;84:106504. https://doi.org/10.1016/j. intimp.2020.106504

17. Cleverley J, Piper J, Jones MM. The role of chest radiography in confirming COVID-19 pneumonia. BMJ. 2020;370:m2426. https://doi.org/10.1136/bmj.m2426

18. Davies NG, Abbott S, Barnard RC, Jarvis Cl, Kucharski AJ, Munday JD, et al. Estimated transmissibility and impact of SARS-CoV-2 lineage B.1.1.7 in England. Science. 2021;372(6538):eabg3055.

https://doi.org/10.1126/science.abg3055

19. Giles B, Meredith P, Robson S, Smith G and Chauhan A. The SARS-CoV-2 B.1.1.7 variant and increased clinical severity-the jury is out. Lancet Infect Dis. 2021;21(9):1213-1214. https://doi.org/10.1016/s1473-3099(21)00356-x

\section{Authors Contribution:}

JHG - Concept and design of the study, prepared first draft of manuscript and manuscript revision; MM - Interpreted the results; reviewed the literature preparation of manuscript and manuscript revision; DPR- Reviewed the literature and manuscript preparation; VT - Concept, Coordination and Statistical Analysis; EAK - Revision of the manuscript.

Work attributed to:

Telangana Institute of Medical Sciences and Research, Hyderabad - 500 032, Telangana, India.

\section{Orcid ID:}

Dr. Jayasri Helen Gali - (D) https://orcid.org/0000-0002-2623-2825

Dr. Manasa Musku - (i) https://orcid.org/0000-0002-3681-5186

Dr. Devireddy Pallavi Reddy - (1) https://orcid.org/0000-0002-2281-6209

Dr. Vimala Thomas - (1) https://orcid.org/0000-0003-2521-8375

Dr. Ehsan Ahmed Khan - (i) https://orcid.org/0000-0001-9435-4675 